\title{
Composición corporal y somatotipo del equipo de gimnasia rítmica, Tricampeón del torneo nacional universitario FENAUDE Chile
}

\author{
Body composition and somatotype of the rhythmic gymnastics team, \\ champion for three years of the national university tournament \\ FENAUDE Chile
}

\begin{abstract}
*Jessica Scarlet Mondaca Urrutia, **Jaime Vásquez Gómez, ***Ricardo Souza de Carvalho \& ${ }^{* * * *}$ César Patricio Faúndez Casanova
\end{abstract}

\begin{abstract}
Mondaca, J. S., Vásquez, J., Souza de Carvalho, R., \& Faúndez, C. P. (2021). Composición corporal y somatotipo del equipo de gimnasia rítmica, Tricampeón del torneo nacional universitario FENAUDE Chile. Revista Ciencias de la Actividad Física UCM, N²2(7), enerojunio, 1-11. DOI: http://doi.org/10.29035/rcaf.22.1.3
\end{abstract}

\begin{abstract}
RESUMEN
El objetivo del presente estudio fue determinar la Composición corporal y somatotipo del equipo de gimnasia rítmica, Tricampeón del torneo nacional universitario FENAUDE Chile. Para el logro de este objetivo se realizó una toma de mediciones antropométricas

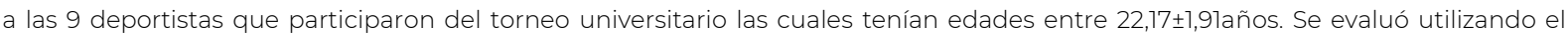
protocolo de marcaje y medición de la International Society for the Avancement in Kine anthropometric (ISAK), y se aplicaron los métodos de Composición Corporal de Deborah Kerr y de Somatotipo de Heath-Carter. Para el análisis estadístico se utilizó el paquete estadístico IBM SPSS V.18 y adicionalmente, para generar la gráfica de la somatocarta se utilizó una planilla realizada en el programa

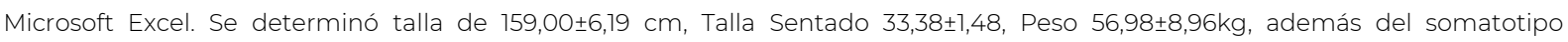
predominante para el grupo de deportistas estudiado es el Endomorfo-Mesomorfo (3,88-3,60-1,78) y la composición corporal, los valores medios fueron: $\mathrm{Mg}=30,42 \%, \mathrm{Mm}=47,12 \% ; \mathrm{Mr}=4,28 \% \mathrm{Mo}=11,68 \%$ y $\mathrm{Mp}=6,51$.
\end{abstract}

Palabras clave: Composición corporal, Antropometría, Somatotipo, Universidad, Gimnasia Rítmica.

\begin{abstract}
The objective of this study was to determine the body composition and somatotype of the rhythmic gymnastics team, three-time champion of the national university tournament FENAUDE Chile. To achieve this objective, anthropometric measurements were taken to the 9 athletes who participated in the university tournament, who were between $22.17 \pm 1.91$ years old. It was evaluated using the marking and measurement protocol of the International Society for the Advancement in Kine anthropometric (ISAK), and the Deborah Kerr Body Composition and Heath-Carter Somatotype methods were applied. For the statistical analysis, the statistical package IBM SPSS V.24 was used and additionally, to generate the graph of the somatochart, a spreadsheet created in the Microsoft Excel program was used. Height of $159.00 \pm 6.19 \mathrm{~cm}$ was determined, Sitting Height $33.38 \pm 1.48$, Weight $56.98 \pm 8.96 \mathrm{~kg}$, in addition to the predominant somatotype for the group of athletes studied is the Endomorph-Mesomorph (3, 88-3.60-1.78) and body composition, the mean values were: $\mathrm{Mg}=30.42 \%, \mathrm{Mm}=47.12 \% ; \mathrm{Mr}=4.28 \% \mathrm{Mo}=11.68 \%$ and $\mathrm{Mp}=6.51$.
\end{abstract}

Key words: Body composition, Anthropometry, Somatotype, College, Rhythmic gymnastics.

\footnotetext{
* Universidad Católica del Maule, Talca, Chile. ID: https://orcid.org/0000-0002-4903-1882jmondaca@ucm.cl

** Centro de Investigación de Estudios Avanzados del Maule (CIEAM), Universidad Católica del Maule, Talca, Chile.

ID: https://orcid.org/0000-0003-0597-793Xjvasquez@ucm.cl

*** Universidad Católica del Maule, Talca, Chile. ID: https://orcid.org/0000-0003-1715-9213 rsouza@ucm.cl

**** Universidad Católica del Maule, Talca, Chile. ID: https://orcid.org/0000-0003-4501-4169 cfaundez@ucm.cl
} 
Mondaca, J. S., Vásquez, J., Souza de Carvalho, R., \& Faúndez, C. P. (2021). Composición corporal y somatotipo del equipo de gimnasia rítmica, Tricampeón del torneo nacional universitario FENAUDE Chile. Revista Ciencias de la Actividad Física UCM, No 22(1), enero-junio, 1-11. DOI: http://doi.org/10.29035/rcaf.22.1.3

\section{INTRODUCCIÓN}

La gimnasia rítmica es un deporte con gran componente estético, de ahí que la composición corporal y el somatotipo sea un factor clave, más aún considerando que en los últimos años se ha vuelto más acrobática con la incorporación de nuevos elementos como saltos y lanzamientos (Aguilera, 2016, et al, 2017, Canda, et al., 2019). Por otra parte, es un deporte que implica importantes demandas físicas y el aprendizaje de elementos técnicos complejos, involucrando capacidades físicas como la fuerza explosiva para el desarrollo de saltos, la coordinación, equilibrio y agilidad para el desarrollo de lanzamientos y destrezas acrobáticas y una gran flexibilidad en toda su ejecución (Vernetta, et al., 2017, Vernetta, et al., 2018).

En este sentido cada especialidad o modalidad deportiva, ya sea individual o colectiva en función de la subespecialización de ciertas funciones tiene un patrón cineantropométrico específico y muy bien definido (Cabañero, et al., 1999, Canda, et al., 2001, Mäestu, et al., 2000) el cual, nos va a permitir conocer cuáles son las características antropométricas que debería tener determinada deportista para alcanzar el éxito deportivo en dicha especialidad. Por ello, tal y como han demostrado diversos estudios (Mäestu, et al., 2000, Moreno, et al., 1996, Pradas, et al., 2013) existe una relación entre el físico del individuo, la modalidad deportiva que practica y el papel de la constitución física como factor de aptitud deportiva, existiendo un claro prototipo físico para lograr un óptimo rendimiento a un alto nivel deportivo. En el caso de la gimnasia rítmica poseer una adecuada composición corporal puede ayudar al aumento de la aceleración y desaceleración de los segmentos corporales durante la ejecución de los gestos técnicos (Conde, et al., 2019, Esper, 2005, Morales, et al., 2018).

Es común encontrar en la mayoría de las modalidades deportivas, de categoría élite, estudios descriptivos de las características antropométricas y de composición corporal (Canda et al., 2019, Franchini, et al., 2011, Milanese, et al., 2011,) sin embargo, los estudios realizados en los últimos 10 años son muy pocos, y sobre todo a nivel universitario (Gil \& Verdoy, 2011, Lima, et al, 2019, Martínez, et al., 2013, Rivera, 2006,). Dado todo lo anterior, y considerando lo planteado por Lima et al. (2019) sobre la baja producción científica en los últimos años sobre la gimnasia rítmica deportiva, principalmente en Chile, el objetivo del estudio es determinar la composición corporal y somatotipo del equipo de gimnasia rítmica, Tricampeón del torneo nacional universitario FENAUDE Chile.

\section{MATERIAL Y MÉTODOS}

Estudio observacional y descriptivo de las características antropométricas, la Composición Corporal y el somatotipo de gimnastas rítmicas universitarias. Se

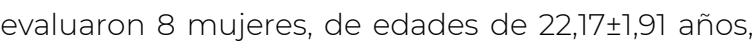
que fueron tricampeonas nacionales del torneo universitario de gimnasia rítmica de Chile, en modalidad conjunto y single durante los años 2015, 2016 y 2017, procedentes de una universidad del centro sur de Chile. La muestra fue determinada mediante procedimiento no probabilístico o por conveniencia, quedando conformada por la totalidad del equipo participante que resulto campeón durante los últimos tres años, sin contar a las deportistas nuevas que participaron solo en la versión 2017. Todas participaron de forma voluntaria, fueron informadas y dieron su consentimiento de acuerdo con la declaración de Helsinki, referentes al anonimato de los nombres y la confidencialidad datos obtenidos (Manzini, 2000).

Para la valoración antropométrica, se siguieron las normas técnicas de medición recomendadas por el International Working Group of Kin anthropometry, según metodología descrita por Ross \& Marfell-Jones en MacDougall et al. (1991) y adoptadas por la International Society for the Advance mentof Kin anthropometry (ISAK, 2011).

Para la toma de muestras se utilizó un kit antropométrico Centurion (Rosscraft Instruments, Canadá), compuesto por cinta métrica Rosscraft Anthrotape, tallimetro Rosscraft, antropómetro Campbell 20, antropómetro Campbell 10, plicómetro 
Mondaca, J. S., Vásquez, J., Souza de Carvalho, R., \& Faúndez, C. P. (2021). Composición corporal y somatotipo del equipo de gimnasia rítmica, Tricampeón del torneo nacional universitario FENAUDE Chile. Revista Ciencias de la Actividad Física UCM, No22(1), enero-junio, 1-11. DOI: http://doi.org/10.29035/rcaf.22.1.3

Gaucho Pro. Se utilizó además un banco antropométrico, una balanza Tanita Ironman (modelo BC-548), y lápiz demográfico. Los datos fueron registrados en planillas de cálculo Excel Antropo gym 2.0.

La recogida de los datos personales necesarios para la valoración antropométrica, la realizó uno de los investigadores, el cual contaba con certificación ISAK nivel 1. Se recogieron los pliegues cutáneos (subescapular, tricipital, bicipital, cresta ilíaca, supra espinal, abdominal, muslo anterior y pierna medial); perímetros (brazo relajado, brazocontraído, muslo frontal y pierna máxima); diámetros óseos pequeños (biepicondíleo del húmero, biestiloideo y bicondíleo del fémur). También se calculó el sumatorio de los 8 pliegues cutáneos. La medición se tomó el día siguiente a la realización del campeonato de gimnasia rítmica universitario en horario de la mañana. Los procedimientos para la recopilación de datos se realizaron siguiendo las recomendaciones técnicas propuestas por la ISAK (Marfell, et al., 2012), considerando el modelo propuesto por Carter \& Honeyman (1990), que contempla el establecimiento del somatotipo en función a coordenadas que determinan un posicionamiento entre los tres estados mórficos; Además, se establecen condicionantes antropomé- tricas en función al fraccionamiento de la masa corporal según lo planteado por Ross \& Kerr (1991). Para determinar el porcentaje de grasa, se utiliza la fórmula para mujeres jóvenes atletas propuesta por Thorland, et al. (1984).

Para el análisis estadístico se utilizó el paquete estadístico IBM SPSS V.18 y, adicionalmente, para generar la gráfica de la somatocarta, se utilizó una planilla realizada en el programa Microsoft Excel. Se ha realizado un análisis descriptivo básico de los datos obtenidos, expresados como media \pm desviación estándar min y máx. para establecer las características antropométricas, así como para determinar el somatotipo.

\section{RESULTADOS}

Las características generales de las gimnastas campeonas nacionales FENAUDE, quedan reflejadas en la Tabla 1. Las gimnastas tenían un promedio de edad de 22,17 años (DE=1,17), el promedio de IMC fue de $22,42 \mathrm{~kg} / \mathrm{m} 2$ (DE=2,01) clasificándose como estado nutricional normal. Por último y de acuerdo a los resultados encontrados se pudo determinar que el índice cintura-cadera presenta un valor de 0,73 $(\mathrm{DE}=0,03)$, lo que las ubica dentro de los parámetros normales.

Tabla 1

Características antropométricas de la muestra

\begin{tabular}{llllll}
\hline & $N$ & $X$ & DS & MIN & MÁX \\
\hline Edad & 8 & 22,17 & 1,91 & 20,24 & 25,42 \\
Peso & 8 & 56,98 & 8,96 & 49,40 & 74,30 \\
Talla & 8 & 159,00 & 6,19 & 151,00 & 170,00 \\
T. Sentado & 8 & 33,38 & 1,48 & 32,00 & 36,00 \\
IMC & 8 & 22,42 & 2,01 & 20,23 & 25,71 \\
ICC & 8 & 0,73 & 0,03 & 0,68 & 0,80 \\
\hline
\end{tabular}

X: promedio; DE: desviación estándar; MIN: mínimo; MAX: máximo

Los resultados de los diámetros, perímetros y pliegues de la muestra aparecen en la Tabla 2, en la cual se puede apreciar sus respectivos promedios y desviaciones estándar para cada indicador 
Mondaca, J. S., Vásquez, J., Souza de Carvalho, R., \& Faúndez, C. P. (2021). Composición corporal y somatotipo del equipo de gimnasia rítmica, Tricampeón del torneo nacional universitario FENAUDE Chile. Revista Ciencias de la Actividad Física UCM, No22(1), enero-junio, 1-11. DOI: http://doi.org/10.29035/rcaf.22.1.3

Tabla 2

Diámetros, perímetros y pliegues de la muestra.

\begin{tabular}{|c|c|c|c|c|c|}
\hline & N & $x$ & DS & MIN & MÁX \\
\hline Tríceps & 8 & 11,75 & 2,66 & 9,00 & 16,00 \\
\hline Subescapular & 8 & 12,13 & 2,30 & 9,00 & 15,00 \\
\hline Bíceps & 8 & 6,75 & 2,12 & 5,00 & 11,00 \\
\hline Cresta ilíaca & 8 & 10,38 & 2,88 & 7,00 & 16,00 \\
\hline Supraespinal & 8 & 11,63 & 2,33 & 8,00 & 15,00 \\
\hline Abdominal & 8 & 12,00 & 2,62 & 9,00 & 15,00 \\
\hline Muslo Frontal & 8 & 11,75 & 4,53 & 5,00 & 18,00 \\
\hline Pantorrilla (máxima) & 8 & 12,13 & 2,75 & 8,00 & 15,00 \\
\hline Cabeza & 8 & 53,98 & 1,58 & 51,00 & 56,00 \\
\hline Cuello & 8 & 30,26 & 1,07 & 29,00 & 32,00 \\
\hline Brazo Relajado & 8 & 23,88 & 1,81 & 22,00 & 27,00 \\
\hline Brazo Flexionado en Tensión & 8 & 26,19 & 2,51 & 23,00 & 31,00 \\
\hline Antebrazo Máximo & 8 & 22,04 & 3,09 & 15,30 & 25,00 \\
\hline Muñeca & 8 & 14,40 &, 85 & 13,40 & 15,50 \\
\hline Tórax Mesoesternal & 8 & 86,13 & 6,86 & 79,00 & 98,00 \\
\hline Cintura (mínima) & 8 & 68,43 & 3,89 & 64,50 & 76,00 \\
\hline Cadera (máximo) & 8 & 93,48 & 6,68 & 87,00 & 104,50 \\
\hline Muslo (máximo) & 8 & 53,75 & 4,23 & 49,00 & 60,00 \\
\hline Muslo (medial) & 8 & 47,88 & 3,53 & 43,00 & 53,00 \\
\hline Pantorrilla (máxima) & 8 & 33,85 & 2,23 & 31,00 & 37,00 \\
\hline Tobillo (mínima) & 8 & 20,54 &, 96 & 19,50 & 22,00 \\
\hline Biacromial & 8 & 36,04 & 1,15 & 34,90 & 37,70 \\
\hline Tórax Transverso & 8 & 26,81 & 1,66 & 24,70 & 29,80 \\
\hline Tórax Antero-posterior & 8 & 26,11 & 1,07 & 25,00 & 28,00 \\
\hline Bi-iliocrestídeo & 8 & 25,84 & 1,56 & 23,60 & 28,10 \\
\hline Humeral (biepicondilar) & 8 & 5,73 &, 29 & 5,40 & 6,10 \\
\hline Femoral (biepicondilar) & 8 & 8,41 &, 41 & 7,70 & 9,10 \\
\hline Muñeca (biestiloideo) & 8 & 4,99 &, 26 & 4,60 & 5,40 \\
\hline Tobillo (bimaleolar) & 8 & 6,49 &, 33 & 6,10 & 7,00 \\
\hline
\end{tabular}

X: promedio; DE: desviación estándar; MIN: mínimo; MAX: máximo 
Mondaca, J. S., Vásquez, J., Souza de Carvalho, R., \& Faúndez, C. P. (2021). Composición corporal y somatotipo del equipo de gimnasia rítmica, Tricampeón del torneo nacional universitario FENAUDE Chile. Revista Ciencias de la Actividad Física UCM, № 22(1), enero-junio, 1-11. DOI: http://doi.org/10.29035/rcaf.22.1.3

En relación a la distribución por tejidos se puede observar que existe una predominancia de masa muscular 46,45\%, seguido de masa grasa 30,93\%. El análisis del somatotipo de todo el grupo, mostro un predomino equilibrado de los componentes muscula- res y graso, aunque con una ligera ventaja del comportamiento muscular sobre el graso, tratándose, por tanto, de un somatotipo Endomorfo-Mesomorfo (3,8 -3,6-1,7), alejado de los puntos hacia el componente ectomórfico, denota características morfológicas robustas. Tabla 3.

TABLA 3:

Composición corporal y somatotipo de la muestra.

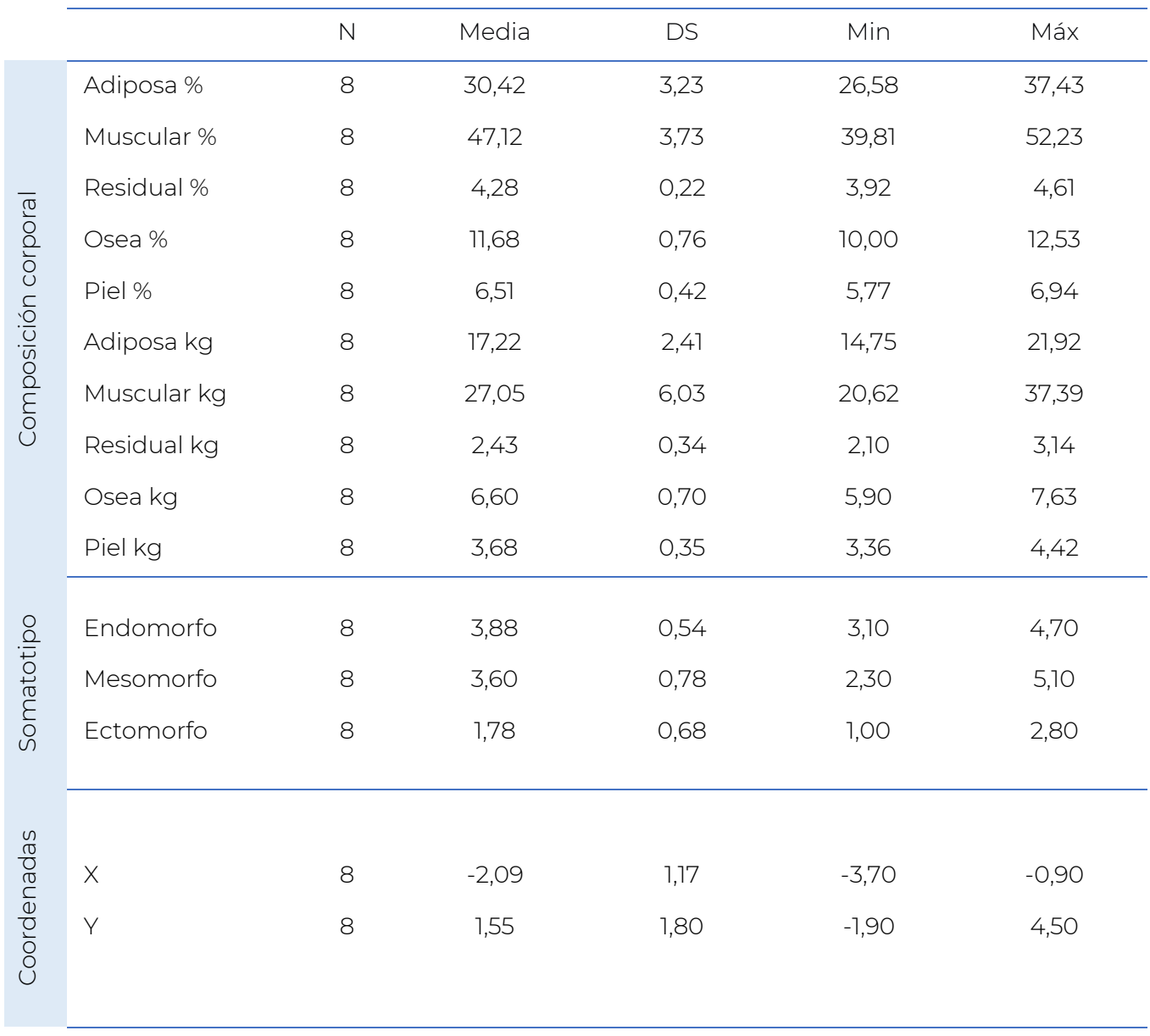

X: promedio; DE: desviación estándar; MIN: mínimo; MAX: máximo 
Mondaca, J. S., Vásquez, J., Souza de Carvalho, R., \& Faúndez, C. P. (2021). Composición corporal y somatotipo del equipo de gimnasia rítmica, Tricampeón del torneo nacional universitario FENAUDE Chile. Revista Ciencias de la Actividad Física UCM, № 22(1), enero-junio, 1-11. DOI: http://doi.org/10.29035/rcaf.22.1.3

La Figura 1 muestra una somatocarta que describe las gimnastas catalogadas de acuerdo al biotipo de individual (puntos rojos) y el punto azul donde se encuentran ubicadas las deportistas evaluadas en este estudio según su media aritmética. Se obtuvo que en promedio (punto azul) tienen características de Endomorfo-Mesomorfo. Lo que nos señala una predominancia hacia la endomorfia en este tipo de deportistas de nivel universitario, las cuales se encuentran lejos del valor ideal para este deporte según el estudio de Menezes et al. (2014), el somatotipo ideal de una gimnasta de elite debe ser Ecto-Mesomorfica, con valores aproximados a 2,3$2,8-4,1$

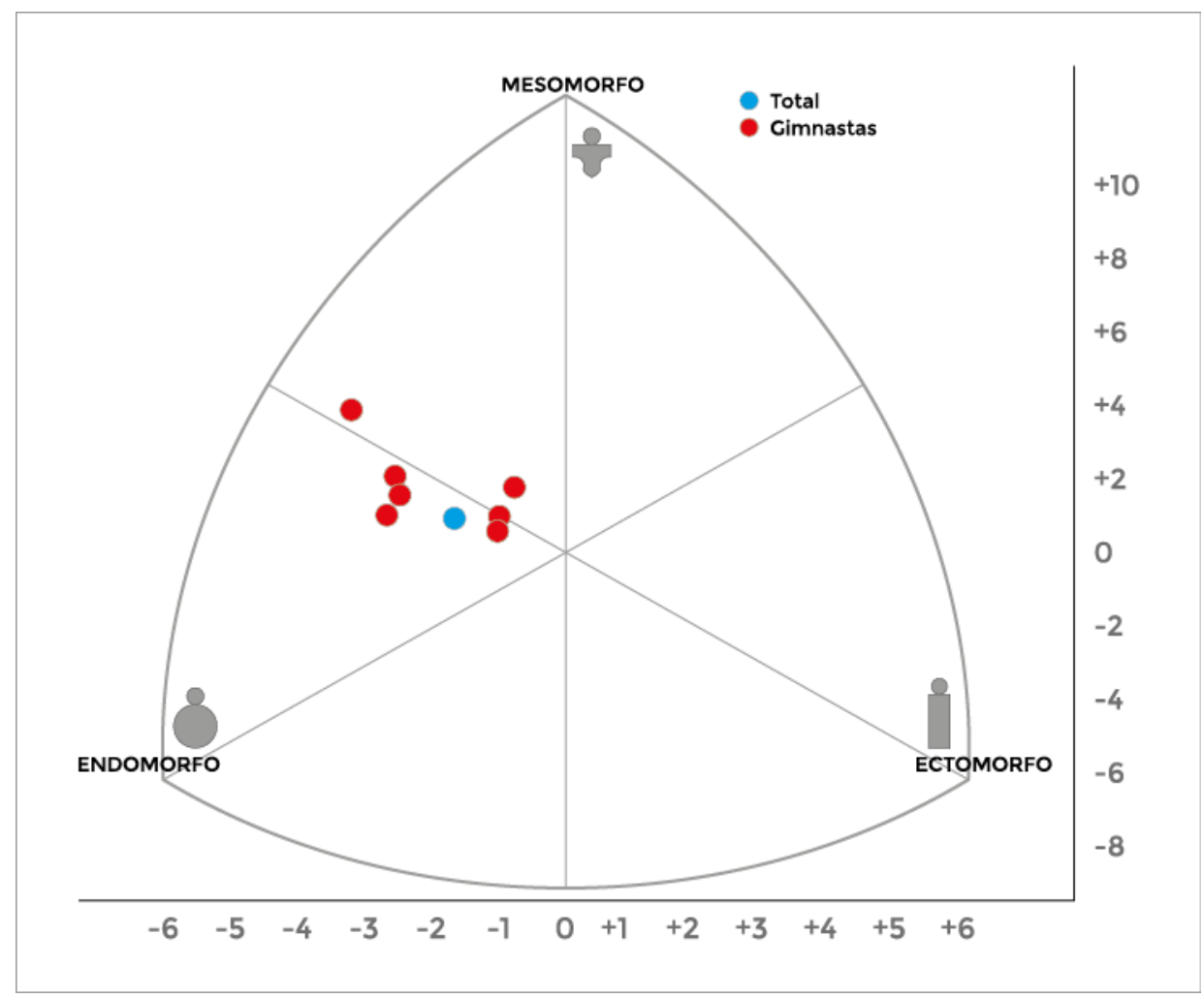

Figura 7. Somatocarta de la muestra. 


\section{DISCUSIÓN}

En este estudio, se definió el perfil antropométrico y los parámetros relacionados con la composición corporal. El análisis de la composición corporal es posible a través de una diversidad de métodos, para ello, es necesario dividir el cuerpo humano en varios componentes medibles (Aguado et al, 2006). En este sentido, una importante herramienta es el fraccionamiento del cuerpo en 5 componentes corporales que permiten analizar y evaluar a niños, adolescentes, jóvenes y adultos. Esta técnica considerada doblemente indirecta(antropometría) fue utilizada para describir la composición corporal de jóvenes universitarios (Cossio, et al., 2011).

Al comparar estos datos con los encontrados en la literatura científica relacionada, cabe señalar que la gimnasia rítmica deportiva, no goza de un gran número de trabajos científicos y menos aún de carácter antropométrico en este grupo de edad (Gimnastas universitarias), lo cual concuerda con lo reportado por Lima et al, (2019). En este sentido, ha sido necesario recurrir a una búsqueda en el extranjero, debido a la escasez de estudios antropométricos existentes de referencia sobre la Gimnasia rítmica deportiva en Chile.

De acuerdo con autores como Menezes et al. (2014) y Poliszczuk \& Broda (2010), la literatura sugiere que la prevalencia de somatotipo y la constitución corporal de gimnastas de alto rendimiento, presentan un perfil Ecto-Mesomorfico lo cual se contrapone con los resultados encontrados en este estudio, ya que las gimnastas universitarias tienen un alto componente Endomorfo.

En cuanto a los resultados encontrados por Irurtia, et al. (2009) en gimnastas españolas desde la infancia hasta la edad adulta, muestran resultados distintos a los de este estudio: Para endomorfia: juveniles 1,5; adultas 1,7; Mesomorfia: juveniles 6.1; adultas 6,3; Ectomorfia: juveniles 2,5; adultas 2,4. En relación a la investigación realizada por Menezes (2006), también encontraron resultados diferentes a este estudio, sobre los valores de cada componente del somatotipo y la constitución corporal que se encuentran en diferentes niveles competitivos de gim-

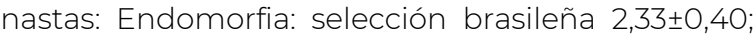

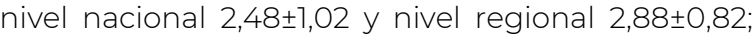

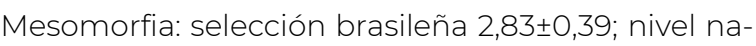
cional $2,81 \pm 1,00$ y nivel regional 3,16 $\pm 0,40$; Ectomor-

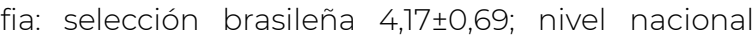

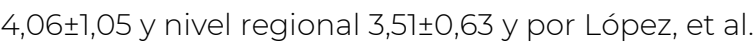
(1991) para endomorfia: 2,3 $\pm 0,45$; Mesomorfia:

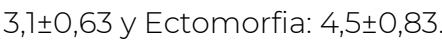

Por su parte, estudios como los de Fernández y Alvero (2006) reportaron datos similares a los de esta investigación, demostrando los siguientes componentes en el somatotipo, Endomorfo-Mesomorfo: 3,6 - 3,9 - 2,5. En el estudio de Romero, et al. (2011) encontraron resultados similares, en gimnastas de categoría Juvenil, en cuanto a su antropometría, Endo-Ectomorfico: 4,4 - 3,3 - 3,5 dejando de manifiesto que las gimnastas a nivel inicial o universitario se alejan mucho del somatotipo ideal para alto rendimiento.

Continuando con el análisis al comparar los resultados de este estudio con la literatura en deportistas universitarios de distintas disciplinas (Almagià, et al., 2008, Bahamondes, et al, 2012, Martinez et al., 2013) se logra evidenciar la predominancia del componente Endomorfo en los deportistas universitarios datos que concuerdan con los encontrados en este estudio. Por último, en un estudio realizado en Chile en sujetos físicamente activos entre 20 y 29 años por Rodríguez, et al. (2010) se encontraron valores similares a los de este estudio (Endomorfo-Mesomorfo: 4,1- 4,2 - 2,1) lo que deja en evidencia la predominancia del componente Endomorfo-Mesomorfo en este grupo de edad, tanto en deportista universitarios como en sujetos físicamente activos y estudiantes universitarios. Sin embargo, menores que en universitarios Chilenos (León, et al., 2012, Vásquez, et al., 2018), en donde el componente endomórfico y el \% de grasa es elevado. 
Mondaca, J. S., Vásquez, J., Souza de Carvalho, R., \& Faúndez, C. P. (2021). Composición corporal y somatotipo del equipo de gimnasia rítmica, Tricampeón del torneo nacional universitario FENAUDE Chile. Revista Ciencias de la Actividad Física UCM, No 22(1), enero-junio, 1-11. DOI: http://doi.org/10.29035/rcaf.22.1.3

Por lo cual, instamos a generar mayor cantidad de datos y variables de distintas disciplinas universitarias para ampliar el universo de comparación y mejorar la referencia comparativa de los deportistas universitarios en Chile.

\section{CONCLUSIONES}

Los resultados de la composición corporal mostraron un mayor porcentaje muscular que graso en las deportistas de gimnasia rítmica deportiva. Con respecto al somatotipo de las campeonas de nacionales de gimnasia rítmica, se ha hallado una predominancia del componente endomórfico, que tiene relación con el alto porcentaje de grasa encontrado, que puede estar influenciado por las características de los estudiantes universitarios. Sin embargo, es importante considerar que el somatotipo no es algo permanente y que puede ser modificado.

A través del presente estudio, realizado con las alumnas universitarias pertenecientes a la selección de gimnasia rítmica deportiva campeona del torneo FEAUDE de la especialidad, se ha podido demostrar que poseen un somatotipo Endomorfo-Mesomorfo con sus resultados de composición corporal, Somatotipo e índices corporales, es una nueva fuente de información de la VII región y actualizada para Chile, la cual favorecerá las comparaciones para futuros estudios, en este y otros deportes, siendo una herramienta adecuada para su utilización.

Teniendo en cuenta los resultados obtenidos de las deportistas de este estudio, se observa que presentan una composición corporal y somatotipo que no favorece el máximo rendimiento para su especialidad deportiva. Esta estructura física en la que no predomina la delgadez con respecto a la adiposidad no favorecerá una fuerza relativa significativa con relación al peso corporal total, esto, por lo tanto, disminuirá la aceleración y desaceleración de los segmentos corporales durante la ejecución de los gestos técnicos.

Al término del articulo cabe resaltar que el equipo evaluado logro un cuarto año seguido como campeón universitario chileno.

\section{BIBLIOGRAFIA}

Aguado, S., Rodríguez, R., \& Gómez, L. (2006). Relación entre composición corporal y edad en sujetos sanos de la Comunidad de Madrid. Revista Científica General José María Córdova, 13(15), 257-270. https://www.redalyc.org/pdf/4762/476247223011.pdf

Castañeda, S., \& Caiaffa, N. (2015). Relación entre la composición corporal y el rendimiento físico en la Escuela Militar de Cadetes José María Córdova. Revista Científica General José María Córdova, 13(15), 257-270. https://www.redalyc.org/pdf/4762/476247223011.pdf

Aguilera, F. L. (2016). Estado nutricional, satisfacción con la imagen corporal e ingesta alimentaria e adolescentes que practican Gimnasia Artística. [Tesis de licenciatura, Universidad FASTA]. REDI - Repositorio Digital - Universidad FASTA. http://redi.ufasta.edu.ar:8080/xmlui/handle $/ 123456789 / 1272$

Almagiá, A., Rodríguez, F., Barraza, F., Lizana, P. J., \& Jorquera, C. (2008). Perfil antropométrico de jugadoras chilenas de futbol femenino. International Journal of Morphology, 26(4), 817-821. http://dx.doi.org/10.4067/S071795022008000400006

Bahamondes, C., Cifuentes, B., Lara, E., \& Berral de la Rosa, F. (2012). Composición corporal y somatotipo en futbol femenino. Campeonato sudamericano Sub-17. International Journal of Morphology, 30(2), 450-460. http://dx.doi.org/10.4067/5071795022012000200016

Barraza, F., \& Rodríguez, F. (2011). Comparación de la Masa Muscular y Masa Grasa de Estudiantes de Primer año de Educación Física. Journal of Movement and Health, 12(1), 34-39. https://dialnet.unirioja.es/servlet/articulo?codigo=3706725 
Mondaca, J. S., Vásquez, J., Souza de Carvalho, R., \& Faúndez, C. P. (2021). Composición corporal y somatotipo del equipo de gimnasia rítmica, Tricampeón del torneo nacional universitario FENAUDE Chile. Revista Ciencias de la Actividad Física UCM, № 22(1), enero-junio, 1-11. DOI: http://doi.org/10.29035/rcaf.22.1.3

Cabañero, M., Canda, A., Bonilla, M., Calderón, C., \& Rubio, S. (1999). Gimnasia rítmica de alta competición: composición corporal y somatotipo. Archivos de Medicina del Deporte, 16, 505-506.

Canda, A., Sainz, L., De Diego, T., de Diego, T., \& Pacheco, J.L. (2001). Características morfológicas del decatleta vs especialistas. Archivos de Medicina del Deporte, 18(84), 277-284. https://pesquisa.bvsalud.org/portal/resource/pt/ibc-23218

Canda, A., Rabadán, M., Sainz, L., \& Agorreta, L. (2019). Evolución del perfil antropométrico y fisiológico de las gimnastas de rítmicas españolas de ejercicios de conjuntos en los Juegos Olímpicos de 1996 y 2016. Revista Andaluza de Medicina del Deporte, 12(3), 258262. https://pesquisa.bvsalud.org/portal/resource/pt/ibc-191861

Carter, J., \& Honeyman, B. (1990). Somatotyping: Development and Applications (Vol. 5). Cambridge University Press.

Conde, H., Caro, W., Chaparro, Y., \& Agudelo, C. (2019). Correlación entre somatotipo y fuerza explosiva de tren inferior de la selección Boyacá de baloncesto masculino, categoría sub 15. VIREF Revista De Educación Física, 8(2), 97-105. https://revistas.udea.edu.co/index.php/viref/article/view/338709

Cossio, M., De Arruda, M., Moyano, A., Gañan, E., Pino, L., \& Lancho, J. (2011). Composición corporal de jóvenes universitarios en relación a la salud. Nutrición Clínica y Dietética Hospitalaria. 37(3), 15-21. https://revista.nutricion.org/PDF/Composicion-corporalCossio.pdf

Esper, A. (2005). Fuerza muscular, composición corporal y saltabilidad. Revista Digital EFDeportes, (88). https://www.efdeportes.com/efd88/fuerza.htm
Franchini, E., Del Vecchio, F., Matsushigue, K., \& Artioli, G. (2011). Physiological profiles of elite judo athletes. Sports Medicine, 47(2), 147166. https://doi.org/10.2165/11538580000000000-00000

Gil Gómez, J., \& Verdoy, P. (2011). Caracterización de deportistas universitarios de fútbol y baloncesto: antropometría y composición corporal. Revista de Ciencias del Deporte, 7(1), 3951.

https://www.redalyc.org/pdf/865/86516191005.pdf

Irurtia, A., Busquets, A., Marina, M., Galilea, P., \& Carrasco, M. (2009). Talla, peso, somatotipo y composición corporal en gimnastas de elite españoles desde la infancia hasta la edad adulta. Apunts Sports Medicine, 44(161), 18$28 . \quad$ https://www.apunts.org/index.php?p=revista\&pii=13135386\&tipo=pdfsimple

ISAK. (2011). International Standards for Anthropometric Assessment. ISAK. https://docplayer.net/25335980-International-standardsfor-anthropometric-assessment.htm

León, H., Torres, A., Arias, I., \& Zea, A. (2012). Análisis del índice de masa corporal, porcentaje de grasa y somatotipo en estudiantes universitarios de primer semestre. Revista de Investigación: Cuerpo, Cultura y Movimiento, 2(3-4), 37-49. https://doi.org/10.15332/s22484418.2012.03-4.02

Lima, L., Moraes, M., Parra, I., Rojo, J., \& do Couto, L. (2019). Gimnasia rítmica: perfil sobre la producción científica en revistas de latinoamérica, caribe y países ibéricos. Pensar en Movimiento: Revista de Ciencias del Ejercicio y la Salud, $\quad 77(2), \quad 24-43$. https://www.scielo.sa.cr/pdf/pem/v17n2/44 2059977004.pdf

López, A., Franco, L., \& Terreros J. (1991). Gimnasia Rítmica. Evolución fisiológica y antropométrica en una temporada. Archivos de Medicina del Deporte, 8(30), 127-133. http://femede.es/documentos/Gimnasia_ritmica_127_30.pdf 
Mondaca, J. S., Vásquez, J., Souza de Carvalho, R., \& Faúndez, C. P. (2021). Composición corporal y somatotipo del equipo de gimnasia rítmica, Tricampeón del torneo nacional universitario FENAUDE Chile. Revista Ciencias de la Actividad Física UCM, № 22(1), enero-junio, 1-11. DOI: http://doi.org/10.29035/rcaf.22.1.3

Mäestu, J., Jürimäe, J., \& Jürimäe, T. (2000). Prediction of rowing performance from selected physiological variables. Differences between lightweight and open class rowers. Medicina dello Spor, 53(3), 247-254. https://www.minervamedica.it/en/journals/medicina-dello-sport/article.php?cod=R26Y2000N03A0247

Manzini, J.L. (2000). Declaración de Helsinki: principios éticos para la investigación médica sobre sujetos humanos. Acta Bioethica, 6(2), 321-334. http://dx.doi.org/10.4067/S1726569×2000000200010

Marfell, M., Stewart, A., \& de Ridder, J. (2012). International standards for anthropometric assessment. International Society for the Advancement of Kinanthropometry. http://hdl.handle.net/11072/1510

Martínez, J., Mielgo-Ayuso, J., \& Janci, J. (2013). Estudio de la composición corporal en deportistas masculinos universitarios de diferentes disciplinas deportivas. Cuadernos de Psicología del Deporte, 12(2), 89-94. https://revistas.um.es/cpd/article/view/177831

Menezes, L., \& Fernandes Filho, J. (2006) Identificação e comparação das características dermatoglíficas, somatotípicas e de qualidades físicas básicas de atletas de GRD de diferentes níveis de qualificação esportiva. Fitness \& Performance Journal, 5(6), 393401.

https://www.redalyc.org/pdf/751/75117270010.pdf

Menezes, L., Novaes, J., \& Fernandes-Filho, J. (2014). Somatotipo de Atletas y Practicantes de Gimnasia Rítmica Prepubescentes y Postpubescentes. International Journal of Morphology, 32(3), 968-972. http://dx.doi.org/10.4067/s071795022014000300036
Milanese, C., Piscitelli, F., Lampis, C., \& Zancanaro, C. (2011). Anthropometry and body composition of female handball players according to competitive level or the playing position. Journal of Sports Science, 29(12), 1301-1309. https://doi.org/10.1080/02640414.2011.59141 9

Morales, J., Palacio, D., \& Molina, S. (2018). Correlación entre la potencia en miembros inferioressuperiores y el porcentaje de masa muscular en nadadores entre 13 a 75 años de la liga de natación de Antioquia. [Tesis de pregrado, Universidad de San Buenaventura]. http://bibliotecadigital.usb.edu.co/bitstream/10819/7021/1/Correlacion_Potencia_Miembros_Morales_2018.pdf

Moreno, J., Camarero, S., \& Tella, V. (1996). Valoración de los parámetros antropométricos en las pruebas de 100 y 200 m mariposa. Apunts: Educación Física y Deportes, (46), 81-86. https://revista-apunts.com/valoracion-delos-parametros-antropometricos-en-laspruebas-de-100-y-200-m-mariposa/

Pradas de la Fuente, F., González, J., Molina, E., \& Castellar, C. (2013). Características Antropométricas, Composición Corporal y Somatotipo de Jugadores de Tenis de Mesa de Alto Nivel. International Journal of Morphology, $37(4)$, 1355-1364. https://dx.doi.org/10.4067/s071795022013000400033

Poliszczuk, T., \& Broda, D. (2010). Somatic constitution and the ability to maintain dynamic body equilibrium in girls practicing rhythmic gymnastics. Pediatric Endocrinology Diabetes Metabolism, 16(2), 94-99. https://pubmed.ncbi.nlm.nih.gov/20813086/ 
Mondaca, J. S., Vásquez, J., Souza de Carvalho, R., \& Faúndez, C. P. (2021). Composición corporal y somatotipo del equipo de gimnasia rítmica, Tricampeón del torneo nacional universitario FENAUDE Chile. Revista Ciencias de la Actividad Física UCM, № 22(1), enero-junio, 1-11. DOI: http://doi.org/10.29035/rcaf.22.1.3

Rivera, J. (2006). Valoración del somatotipo y proporcionalidad de futbolistas universitarios mexicanos respecto a futbolistas profesionales. Revista Internacional de Medicina y Ciencias de la Actividad Física y del Deporte, 6(21), 16-28. http://hdl.handle.net/10486/3646

Rodríguez, F., Almagià, A., Yuing, T., Binvignat, O., \& Lizana, P. (2010). Composición corporal y somatotipo referencial de sujetos físicamente activos. International Journal of Morphology, 28(4), 1159-1165. http://dx.doi.org/10.4067/S071795022010000400028

Romero, B., Palomino, A., \& González, J. (2011). El perfil antropométrico de la gimnasia rítmica. Apunts. Educación Física y Deportes, (103), 48-55. https://revista-apunts.com/el-perfilantropometrico-de-la-gimnasia-ritmica/

Ross, W., \& Kerr, D. (1991). Fraccionamiento de la masa corporal: un nuevo método para utilizar en nutrición clínica y medicina deportiva. Apunts Sportes Medicine, 28(109), 175188. https://www.apunts.org/en-fraccionamiento-masa-corporal-un-nuevo-articulox0213371791052237

Taboada, Y., Vernetta, M., \& Gutiérrez, Á. (2017). Anthropometric Profile in Different Event Categories of Acrobatic Gymnastics. Journal of Human Kinetics, 57, 169-179. https://www.ncbi.nlm.nih.gov/pmc/articles/PMC5504589/

\section{Dirección para correspondencia}

\section{Mg. Jéssica Scarlet Mondaca Urrutia}

Universidad Católica del Maule

Talca, Chile.

Dirección postal: Avenida San Miguel, 3605 CP: 3460000

ORCID: https://orcid.org/0000-0002-4903-1882

Contacto:

jmondaca@ucm.cl

Recibido: 28-10-2020

Aceptado: 28-01-2021
Thorland, W., Johnson, G., Tarp, G., Fagot, T., \& Hammer, R. (1984). Validity of anthropometric equations for the estimation of body density in adolescent athletes. Medicine and Science in Sports and Exercise, 16(1), 77-81. https://pubmed.ncbi.nlm.nih.gov/6708784/

Vásquez, J., Castillo, M., Souza de Carvalho, R., Faúndez, C., \& Torrealba, A. (2018). Antropometría, nivel de actividad física y condición física en estudiantes de educación física tras cuatro años en la universidad. Nutrición Clínica y Dietética Hospitalaria, 38(1), 160-164. https://doi.org/10.12873/381JVasquez

Vernetta, M., Montosa, I., Beas, J., \& López, J. (2017). Batería Funcional ARISTO en Gimnasia Rítmica: protocolo de test específicos para la evaluación de jóvenes gimnastas en un ámbito de entrenamiento saludable. Revista Andaluza de Medicina del Deporte, 10(3),

112-119. https://dx.doi.org/10.1016/j.ramd.2017.02.001

Vernetta, M., Montosa, I., \& Peláez, E. (2018). Estima corporal en gimnastas adolescentes de dos disciplinas coreográficas: gimnasia rítmica y gimnasia acrobática. Psychology, Society \& Education, 10(3), 301-314. http://dx.doi.org/10.25115/psye.v10i3.2216 